\title{
DNNARA: A Deep Neural Network Accelerator using Residue Arithmetic and Integrated Photonics
}

\author{
Jiaxin Peng \\ vitapp@gwu.edu \\ The George Washington University \\ Washington D.C
}

\author{
Yousra Alkabani \\ yousra.alkabani@hh.se \\ Halmstad University \\ Halmstad, Sweden
}

\author{
Shuai Sun \\ sunshuai@gwu.edu \\ The George Washington University \\ Washington D.C
}

\author{
Volker J. Sorger \\ sorger@gwu.edu \\ The George Washington University \\ Washington D.C
}

\author{
Tarek El-Ghazawi \\ tarek@gwu.edu \\ The George Washington University \\ Washington D.C
}

\begin{abstract}
Deep Neural Networks (DNNs) are currently used in many fields, including critical real-time applications. Due to its compute-intensive nature, speeding up DNNs has become an important topic in current research. We propose a hybrid opto-electronic computing architecture targeting the acceleration of DNNs based on the residue number system (RNS). In this novel architecture, we combine the use of Wavelength Division Multiplexing (WDM) and RNS for efficient execution. WDM is used to enable a high level of parallelism while reducing the number of optical components needed to decrease the area of the accelerator. Moreover, RNS is used to generate optical components with short optical critical paths. In addition to speed, this has the advantage of lowering the optical losses and reducing the need for high laser power. Our RNS compute modules use one-hot encoding and thus enable fast switching between the electrical and optical domains.

In this work, we demonstrate how to implement the different DNN computational kernels using WDM-enabled RNS based integrated photonics. We provide an accelerator architecture that uses our designed components and perform design space exploration to select efficient architecture parameters. Compared to memristor crossbars, our residue matrix-vector multiplication unit has two orders of magnitude higher peak performance. Our experimental evaluation using DNN benchmarks illustrates that our architecture can perform more than 19 times faster than the state of the art GPUs under the same power budget.
\end{abstract}

\section{CCS CONCEPTS}

- Computer systems organization $\rightarrow$ Neural networks; Optical computing; • Computing methodologies $\rightarrow$ Parallel computing methodologies.

Permission to make digital or hard copies of all or part of this work for personal or classroom use is granted without fee provided that copies are not made or distributed for profit or commercial advantage and that copies bear this notice and the full citation on the first page. Copyrights for components of this work owned by others than ACM must be honored. Abstracting with credit is permitted. To copy otherwise, or republish, to post on servers or to redistribute to lists, requires prior specific permission and/or a fee. Request permissions from permissions@acm.org.

ICPP '20, August 17-20, 2020, Edmonton, AB, Canada

(C) 2020 Association for Computing Machinery.

ACM ISBN 978-1-4503-8816-0/20/08 . \$ \$15.00

https://doi.org/10.1145/3404397.3404467

\section{KEYWORDS}

residue number system, deep learning, neural network accelerators, optical computing

ACM Reference Format:

Jiaxin Peng, Yousra Alkabani, Shuai Sun, Volker J. Sorger, and Tarek ElGhazawi. 2020. DNNARA: A Deep Neural Network Accelerator using Residue Arithmetic and Integrated Photonics. In 49th International Conference on Parallel Processing - ICPP (ICPP '20), August 17-20, 2020, Edmonton, AB, Canada. ACM, New York, NY, USA, 11 pages. https://doi.org/10.1145/3404397.3404467

\section{INTRODUCTION}

Deep Neural Networks (DNNs) are one of the critical methods of machine learning, which are widely used in modern applications, such as image classification, real-time automotive, health care, and military applications [43, 44]. A neural network consists of several layers between the input and the output, aiming to learn different features and classify the output into different classes. In this paper, we target the acceleration of Convolutional Neural Networks (CNNs) that represent one of the most popular classes of DNNs. A CNN is typically composed of groups of convolutional and pooling layers to support feature learning, followed by fully connected layers for classification.

Matrix-vector multiplication (MVM) is the main computational kernel in CNNs. The first block in Figure 1 shows an example of MVM, where the matrix size is $3 \times 3$, and the vector size is $3 \times 1$. The output of this operation is a $3 \times 1$ vector whose elements $c_{i}=\sum_{j} a_{i j} b_{j}$ are computed using three independent multiplyaccumulate (MAC) operations. The efficiency of any CNN accelerator depends on its ability to (1) execute multiple MVM operations at high-speed, and (2) map the data to the architecture in order to minimize data movement. For instance, NVIDIA GPUs mainly depend on having a large number of CUDA cores and tensor cores combined with a hierarchy of high-bandwidth memories [17]. Multiple FPGA-based CNN accelerators depend on using a network of hardware MACs $[1,2,36,60]$.

Recently multiple accelerators that depend on emerging technologies have also been proposed with the core idea of speeding up the MVM operations. The most notable of such technologies are those based on non-volatile memories (NVMs) and photonic architectures [58]. One of the most promising NVM technologies used for DNN acceleration is memristors [6, 37, 47]. At the core of 


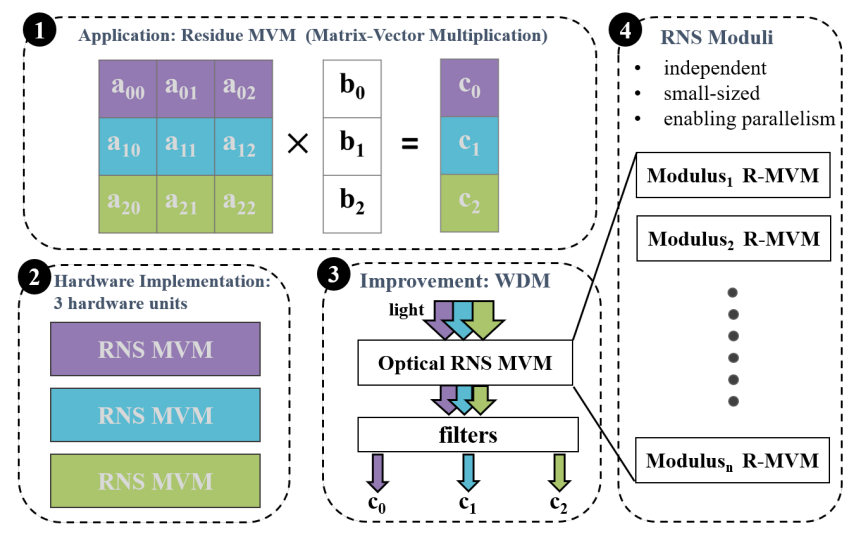

Figure 1: MVM using WDM-enabled Optical RNS MAC.

every memristor-based accelerator lies a crossbar that implements an analog MAC. While a memristor crossbar brings processing closer to memory and speeds up MAC operations relative to digital implementation, the speed of a memristor crossbar is slow $(\sim 100 n s)$ [47] compared to the speed an optical MAC can reach. On the other hand, photonics can achieve superior speeds that can be close to the speed of propagation of light in the used material; however, the current technology suffers from a large area and impractically laser power requirement as the size of the optical critical path increases.

In our work, we introduce a novel DNN accelerator using residue arithmetic (DNNARA), based on integrated photonics. It attempts to provide a practical accelerator from both the area and the power. While we hope that as the technology matures, more compact photonic structures will be available, we believe that we can still benefit from this high-speed technology at its current state. We attempt to reduce the area of our chip while maintaining a high level of parallelism using Wavelength Division Multiplexing (WDM)-enabled MACs combined with filters, as illustrated in block 3 in Figure 1. In this example, we execute the three independent MACs using one optical MAC, where each row is input to the same MAC using different frequencies. At the output, different results are extracted using filters.

In order to have a power-efficient chip while maintaining scalability, we attempt to reduce the optical critical path by using a Residue Number System (RNS)-based implementation of our system. RNS has been used to speed up computations in multiple applications, including cryptography $[3,22]$ and machine learning $[35,45]$ in the electronic domain. In RNS, a number is represented as a set of smaller numbers (residue set) for pairwise co-prime moduli. It exposes the high potential for parallelism because residue arithmetic is digit-irrelevant for both addition and multiplication. For each residue, all operations could be executed independently of the other residues, and the results only need to be combined at the end. The use of RNS computations has two main advantages: (1) using smaller optical MACs with shorter critical paths that are power efficient as the optical losses are minimized through the circuit, and (2) numbers in our system are represented using one-hot encoding, and this relieves the need of using DACs/ADCs, as will be explained in later sections.

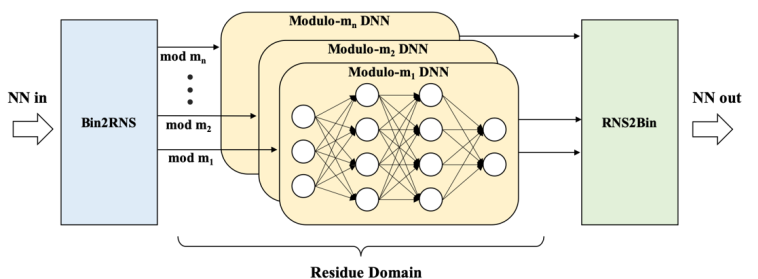

Figure 2: Block Diagram of a DNNARA System.

Figure 2 shows a high-level representation of a DNNARA chip. DNNARA is not a pure optical accelerator but is rather a hybrid opto-electrical system. The transition between the optical and the electrical domain is done seamlessly using high-speed photodetectors and on-chip laser sources. Each chip includes a hierarchy of high bandwidth memories to store and distribute data. The input of the chip takes binary data and converts them to the RNS domain. All the computations through the neural network are done in the RNS domain. Thus, we have the equivalent of multiple small-sized CNNs being executed. We exit the RNS domain only when the output of the CNN is reached. This has the advantage of saving the overhead of converting to/from the RNS domain. Our experimental evaluation shows at least an order of magnitude performance improvement over other technologies when using $\mathrm{CNN}$ benchmarks and running the system at the same power budget.

\section{BACKGROUND}

\subsection{Convolutional Neural Network}

A convolutional neural network is widely used in classification, e.g., image recognition, by utilizing different layers, including convolutional layers, fully connected layers, and max pooling layers. Activation functions may happen after some layers, adding the nonlinearity for the results. Within all the layers, MAC operation, sigmoid/hyperbolic tangent function, and maximum function are the main computational kernels considered in this work.

Multiply-accumulate operations are the most computationally intensive parts in a convolutional layer, as well as a fully connected layer, up to billion ones (e.g. VGG [49] and GoogLeNet [54]) in a neural network [5], and occupy more than $90 \%$ computation time [15]. Activation function comes after the accumulation with a bias to add nonlinearity. ReLu, Sigmoid $(\sigma)$ function and hyperbolic tangent function (tanh) are some typical non-linear activation functions. In our work, the sigmoid function is selected as the activation function. However, it would be hard to calculate in the residue domain. Thus, Taylor series would be applied to the $\sigma$ function. Max pooling layers downsample the previous convolutional layer's output by producing the maximum value out of $n^{2}$ outputs when using an $n \times n$ kernel. Therefore, the maximum function unit is exploited for the max pooling layers.

\subsection{Residue Number System}

A residue number system represents a number $X$ as one set of remainders $\left\{r_{1}, r_{2}, \ldots, r_{n}\right\}$ for selected set of moduli $\left\{m_{1}, m_{2}, \ldots, m_{n}\right\}$, where $r=|X|_{m}$. All the designated moduli should be relatively prime to each other. Primes, therefore, are typically chosen as the 
moduli in such system. The notation of number $X$ in RNS could be represented as $X=\left\{r_{1}, r_{2}, \ldots, r_{n}\right\}_{\left[m_{1}, m_{2}, \ldots, m_{n}\right]}$. For example, if the selected modulus set is $\{2,3,5,7\}$, then the representation of number $\mathrm{X}$ equals to 20 would be $\{0,2,0,6\}_{[2,3,5,7]}$. The representation in RNS, named as dynamic range, could be ranged from 0 to $M-1$ for an unsigned number, where $M=\prod m_{i}$ for all the selected moduli.

A negative integer could be represented in RNS as well, which is similar to the two's complement in a binary system. The complement in a residue system is related to the modulo size, and thus the remainder could be noted as $r=|m-|-\left.\left.X\right|_{m}\right|_{m}$, where X is negative. A negative number, -20 , for instance, could be represented as $\{2-0,3-2,5-0,7-6\}_{[2,3,5,7]}=\{0,1,0,1\}_{[2,3,5,7]}$. The dynamic range would be either $[-(M-1) / 2,(M-1) / 2]$ if $M$ is odd, or $[-M / 2, M / 2-1]$ if $M$ is even for a signed residue system.

Moduli set selection influences the whole system design since it is the fundamental part of a system. There is only one property that should be satisfied in moduli set selection, that is, all moduli should be relatively prime. All prime is selected as our moduli set. In proposed work, electro-optical $2 \times 2$ switches [52] are chosen to implement the residue computation blocks. As an active control component, light loss existed for all possible states, which limits the size of the residue adders/multipliers. Here is a special set $\left\{2^{n}-1,2^{n}, 2^{n}+1\right\}$ and its extensions is one of the most popular choices since it is more hardware friendly to binary conventional systems [39, 45]. However, it is not suitable for nanophotonic design. Recent researches [7, 8, 42] implemented optical residue computer by utilizing optical switch. In such nanophotonic design, the more switches the light propagates, the more laser power and area are required. As the modulo size increases, the hardware demand will not be suitable anymore for the special set, $\left\{2^{n}-1,2^{n}, 2^{n}+1\right\}$. On the other hand, all prime moduli set reduces the critical optical path and requires less energy, and thus, it has been chosen in proposed work.

The arithmetic in residue number system is modulus independent, which is the fundamental property that allows the potential of parallel computing. That is, each digit could be operated on separately without affecting other digits. For example, if $\mathrm{X}=20$ and $\mathrm{Y}=5$, then $X+Y$ could be calculated as $\{0,2,0,6\}_{[2,3,5,7]}+\{1,2,0,5\}_{[2,3,5,7]}$ $=\{0+1,2+2,0+0,6+5\}_{[2,3,5,7]}=\{1,1,0,4\}_{[2,3,5,7]}$. The result of $20+5$ is 25 , which is represented exactly the same format. As long as the final result is within the dynamic range, the internal overflow of the calculation could be ignored. The same rule applies to residue subtraction and multiplication as well.

\section{RELATED WORK}

Many research works show the importance of accelerating the deep neural networks due to its compute-intensive property. The accelerators could be built based on both conventional CMOS technologies and emerging technologies. Several CMOS-based platforms DNN accelerators are available including GPUs [14, 21], FPGAs [19, 30, 61], ASIC [11, 32] and TPUs [27]. The performance of CNN/DNN is limited by the intensive computation and data movement nature of DNNs.

Photonics and analog-based processing-in-memory have emerged as alternative technologies. Resistive random access memory (ReRAM) crossbars $[13,47,50]$ are one of the most popular choices as the near-memory technology, which perform the calculation based on Kirchoff's Law. It provides high data parallelism and combines a hybrid digital-analog computing system. Analog-to-digital converters (ADCs) and digital-to-analog converters (DACs) are required for such a system. The main drawback is the long crossbar read/write time, which constrains the computing throughput. To avoid the conversions, [26] implemented an architecture that maintained all the computations in the digital domain and thus achieved higher speedup and lowers energy requirement.

Optical technologies faced the same problem if a hybrid electrooptic computing system is utilized. Optical-electrical and electricaloptical (OE/EO) conversions will be the additional overhead for a photonic-based accelerator; however, the computations could be executed at light speed and low power consumption. [23] proposed a low energy MAC unit by encoding both inputs as optical signals. By detecting the phase changes, the results would be derived. However, to perform the non-linear functions, conversion to an electrical signal is required. All-optical computer for machine learning is proposed in [31]. Nevertheless, it could not be fit as a chip-size accelerator and is not compatible with current electrical digital computers, resulting in a standalone all-optical machine. Instead, HolyLight [33] proposed two types of architecture as a neural network at the chip-level size. HolyLight-M runs at highspeed $(12.8 \mathrm{GHz})$ compared to a normal electrical circuit, which might cause inconsistency. In addition, laser power is not considered, which is the major energy consumption in an electro-optical design. It increases exponentially as the size of the optical circuit increases.

RNS was used to develop deep learning accelerations, such as CNNs, for its high data parallelism, resulting from its carry-free arithmetic. [35] implemented an FPGA-based accelerator for CNNs, using RNS as the fundamental arithmetic and LUTs as the computation strategy. To alleviate the requirement of LUTs, nested RNS was proposed, which utilizes smaller moduli set to represent the number of larger modulus, compared to regular RNS. Hence, the hardware could be re-utilized. In addition, [4] proposed ROM-based accelerator for CNNs and [38] emphasized the multiplier for neural network acceleration. They are all implemented on FPGAs. RNSnet [45] is proposed to perform all the operations in memory without converting it to the analog domain. It reached high efficiency by decomposing the residue multiplication to local residue additions instead. To the best of our knowledge, the proposed DNNARA architecture is the first one based on RNS as the CNN accelerator with photonic technology.

\section{OVERVIEW OF A DNNARA}

The proposed accelerator, DNNARA, is built based on residue arithmetic and integrated photonics, as shown in Figure 3. The proposed DNNARA chip is built with several tiles, and each tile consists of several residue MVM (R-MVM) units. Furthermore, it comes with additional hardware support, including binary-to-residue (bin2RNS) converters, residue-to-binary (RNS2bin) converters, activation function units, max pooling units, LUTs that store the switch states and input/output information, eDRAMs that store the data information, one additional R-Accumulator unit that could accumulate the 
results from different tiles or R-MVM units, as well as a bus that connects all the units.

\begin{tabular}{|c|c|c|c|}
\multicolumn{3}{|c}{ Chip } & \\
\hline $\mathrm{T}$ & $\mathrm{T}$ & $\mathrm{T}$ & $\mathrm{T}$ \\
\hline $\mathrm{T}$ & $\mathrm{T}$ & $\mathrm{T}$ & $\mathrm{T}$ \\
\hline $\mathrm{T}$ & $\mathrm{T}$ & $\mathrm{T}$ & $\mathrm{T}$ \\
\hline $\mathrm{T}$ & $\mathrm{T}$ & $\mathrm{T}$ & $\mathrm{T}$ \\
\hline $\mathrm{RNN} 2$ Bin & & \multicolumn{1}{|c|}{ Bin2RNS } \\
\hline
\end{tabular}
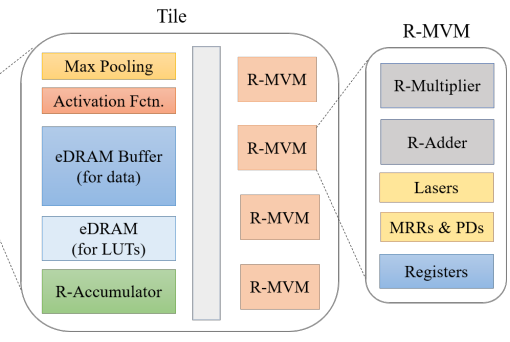

Figure 3: Overview of DNNARA

Bin2RNS and RNS2bin units are required for the conversion between the conventional binary system and the residue number system. A binary number will be converted to residue number via the bin2RNS unit before storing it in the eDRAM buffer. Then all the computation would be performed in the residue domain. Finally, once all the operations are executed, the results will be converted back to the binary domain via the RNS2bin units. The bin2RNS unit is adopted from [55] that implemented this conversion with optical residue adders by setting up the coefficient as the binary information. The RNS2Bin unit is adopted from [53] by converting RNS to the weighted number system before deriving the binary numbers.

Applying RNS to a CNN/DNN is beneficial from its high parallel property, especially for the MAC operations. Thus, we try to avoid the conversions between binary and residue number system. However, activation functions, e.g., sigmoid and hyper tangent functions, are hard to be implemented with RNS. Thus, Taylor series would be applied to the $\sigma$ and tanh function. RNSnet [45] has been studied the accuracy of CNN in RNS. The data width with more than 16-bit could reach $0 \%$ quality loss compared to the baseline of different types. In addition, the Taylor series for activation function is proposed to avoid multiple conversions between binary systems and residue systems. Taylor series could be considered as a polynomial and could be implemented with specialized adders and multipliers, which will be discussed in Section 5.3. Though the proposed design focuses on sigmoid as the activation function, another popular one, Rectified Linear Unit (ReLU), is supported in RNS as well.

Max-pooing layers downsample the output by extracting the maximum value in a kernel. It is crucial to building a comparator or sign detector for such a layer since the sign representation in residue arithmetic is implicit. A sign detector that exploits the conversion from RNS to mixed-radix number system has been proposed [53], which could be implemented with residue adders and multipliers. The detailed of the residue max pooling unit is discussed in Section 5.5. The R-Accumulator is designed to sum the partial results that scheduled for different R-MVMs or tiles.

The R-MVM unit performs the MAC operations in the residue domain. It consists of optical components in order to take advantage of the ultra-fast computation process in the optical domain with low power consumption. AS-Benes residue adders and multipliers are the primary building components. Selected R-Adders and R-Multipliers require look-up tables (LUT) that store switch states for different operations. The LUTs are located in the eDRAM. Lasers are required as the light source, and photo-detectors (PDs) are needed as the optical-electrical (OE) converter. Microring resonators (MRRs) drop light into the PDs as long as the frequency of the light within the designated spectrum. Detailed design will be discussed in Section 5.2.

\section{INTEGRATED PHOTONIC RESIDUE ARITHMETIC COMPUTING ENGINE FOR NEURAL NETWORK}

A neural network contains massive MAC operations, and thus the MVM unit is considered as one of the essential components for a neural network. In this section, we explore the possibility of implementing a residue MVM unit which performs MAC operations in integrated photonic technology. Sigmoid activation function units and max pooling units will be implemented as well. In addition, the performance of the MAC unit benefits from the wavelengthdivision multiplexing (WDM) feature of the nanophotonic device.

\subsection{Integrated Photonic Residue Adder and Multiplier}

Residue adders (R-Adders) and multipliers (R-Multipliers) are the main components in an R-MVM unit, which could be implemented by nanophotonic technology with high-speed and low power consumption. An optical $2 \times 2$ switch was proposed as the essential device to implement the residue adder and multiplier [7, 8, 25, 42, 55]. Figure 4 (a) and (b) depict two states of an electro-optical $2 \times 2$ switch with different values of the electrical control signal. The light either passes through or cross the switch by setting up the electrical control signal to different voltages. Work [25] considered residue operations as maps. For all possible inputs, it will be mapped to the same value but with different arrangements. A non-blocking network could implement this design. Arbitrary-size Benes (ASBenes) network [10], illustrated in Figure 4 (c) and (d), has been shown in work [41] that it requires less optical switches and is being chosen as the residue adders in this work. The AS-Benes scheme allows N-to-N nonblocking communication. It is developed based on regular Benes scheme [9] where $\mathrm{N}$ equals to $2^{n}$ ( $n$ is an integer). However, in the proposed DNNARA architecture, the moduli set are all primes, which does not equal to $2^{n}$ (except for 2). An arbitrary-size scheme breaks this rule and requires fewer switches. For example, a modulo-5 adder will be mapped to an 8-to-8 Benes network and mapped to a 5-to-5 AS-Benes network. Therefore, the area and power overhead decrease. The overall demand of the $2 \times 2$ switches of an AS-Benes network, $S(n)$, could be calculated as $S(N)=2\left\lfloor\frac{N}{2}\right\rfloor+S\left(\left\lceil\frac{N}{2}\right\rceil\right)+S\left(\left\lfloor\frac{N}{2}\right\rfloor\right)$, where $S(2)=1$ and $S(3)=3$.

The logic for the AS-Benes residue adder is built based on the routing capability for the system. A lookup table (LUT) is needed to store the desired switch states required for different computations. By loading the states from the LUT and setting up the switches accordingly, the input light could be routed to the target position. The switches states of all possible operands will be pre-calculated and stored in the LUT by using the division algorithm [28]. This algorithm claims that it is the optimal one to route a Benes network form outermost stages to the central one. Once the states are determined, it will be stored in the LUT and will not be changed anymore. 
When an operation is being executed, the pre-calculated states will be loaded accordingly. For instance, example path shown in Fig.4 (e) illustrates how the light will be propagate in an AS-Benes modulo-5 adder, with respect to operation $|3+4|_{5}=2$. First, operand " $+4 "$ will be encoded, and all the switches will be set to corresponding states after loading them from the LUT. "CCBCCBBB" (states for S1 S8) are the states in this example, where $C$ and $B$ represent the cross and bar state, respectively. Then After loading and setting up the states accordingly, the laser will inject light at input port 3. The input light will pass S2, S3, and S8, while the S2 is at cross-state, and S3, as well as S8, are at bar state. Finally, it will be detected by the photo-detector at output port 2. It is worth to note that, once the states are set as " +4 ", the light at any input port will be routed the expected outputs without reconfiguring the switches. Thus, a small LUT $(<256 \mathrm{~B})$ is enough for a residue adder with chosen 16-bit data width, which could be read within one clock cycle from the simulation result. LUTs are often utilized in traditional RNS computers, storing the pre-calculated results. However, the LUT size would be relatively large compared to storing the states only in our proposed design. (a)

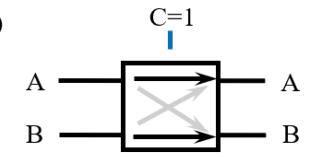

(c)

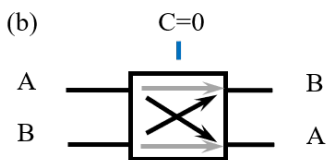

(e)

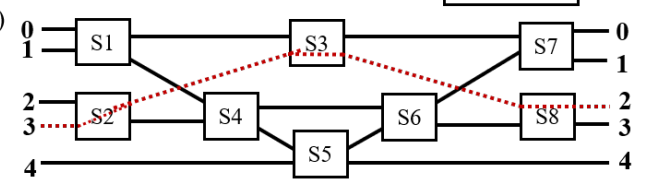

(f)

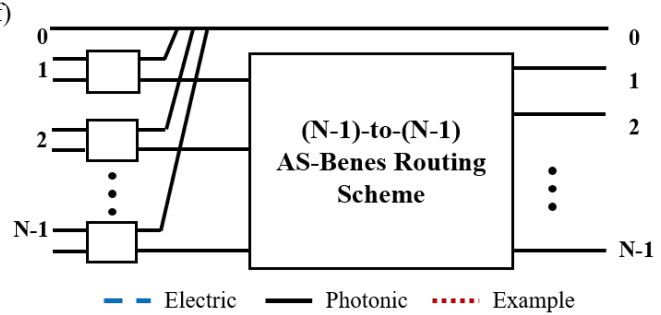

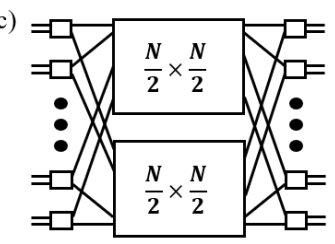

Figure 4: Residue Adder Schematic. An electro-optical $2 \times 2$ switch in (a) bar state and (b) cross state. Schematic of an AS-Benes residue adder if the input is (c) even or (d) odd. (e) An AS-Benes modulo-5 adder. (f) A Modulo-N Residue Multiplier Implementation.

The design of the modulo-N multiplier follows the same idea of residue adder except for multiplication by zero, illustrated in Figure 4 (f). The result of a multiply operation with zero would always be zero, hence the architecture of the residue multiplier should stick to this situation. The rest could be considered as a $(\mathrm{N}-1)$-to-(N-1) routing system, following the map rule in residue arithmetic [25]. Moreover, this method guarantees that the time of residue multiplication is the same as the time of residue addition. Residue computer systems benefit from the fast speed of multiply operations.

\subsection{Wavelength Division Multiplexing Capable Residue MVM Computing Block}

A residue MVM (R-MVM) unit executes the MAC operations, which could be implemented with R-Adders and R-Multipliers. Besides, the wavelength division multiplexing (WDM) capable of selected photonic devices allows several operations executing simultaneously. The following example shows how an R-MVM unit performs several MAC operations in a neural network using WDM.

Assuming here are a $5 \times 5 \times 1$ input feature and a $2 \times 2$ kernel, with stride size one and zero padding (Figure 5 (a)). The convolution of the first two columns of the input feature with the kernel will be mapped to a matrix-vector multiplication and addition (MVMA), $Y=X \times W+B$, as shown in Figure 5 (b). The matrix $\mathbf{X}$ is not the same as the input feature. It is rearranged according to the kernel size. Vector $\mathbf{B}$ represents the bias value. Vector $\mathbf{W}$ is set as the electrical control signal for the multipliers, and matrix $\mathbf{X}$ will be encoded as an optical signal. For instance, $w_{0}$ will be treated as the select signal to load the corresponding switch states from the LUT. The R-Multiplier will be set up accordingly. Then the first row of matrix X will be encoded as a light signal for four R-Multipliers. By utilizing the WDM feature, the first column of $\mathrm{X}\left(x_{0}, x_{1}, x_{2}, x_{3}\right)$ will be represented by different wavelengths, $\lambda_{0}, \lambda_{1}, \lambda_{2}, \lambda_{3}$, respectively. They will be injected into corresponding input ports. Several sets of micro-ring resonators (MRRs) filter out the light with designated wavelengths. The photo-detector (PD) set after each MRR will detect the light. If the light passes through the same waveguide with other wavelengths, it will propagate through this MRR with low loss until it reaches the MRR with designated resonance frequency. For example, the first MRR is designed for $\lambda_{0}$, and the PD will detect only the light at the same wavelength. Therefore, based on the signal of the PD, the result of the operation would be derived. Note that each row will be encoded as the same wavelength for all the multipliers, which decreases the complexity of the hardware. After detecting the multiplication results, optical signals would be converted to electrical signals and are used to set the states for the switches inside a group of residue adders. These sets of adders will sum the multiplication results. Light will be injected again based on the bias in this case. Then the result, $y_{i}$, would be stored in registers.

The size of matrix X should be more extensive in real applications. Due to the area/power constraints, there will not be unlimited resources in an R-MVM. To further explore the opportunity of a residue MAC operation for large size vector, matrix-vector multiplication is split into smaller sizes, which could fit in a single R-MVM unit. Then, sub-operations could be executed in other R-MVM units before accumulating all multiplied results. Registers would be required to store the partial results. The R-Accumulator in the tile will accumulate the partial sum that distributed into different RMVMs. Moreover, it is hard to support too many wavelengths in such a system. We limit our design to a small number of frequencies 
(WDM size), $3 \sim 5$, resulting in the necessity to decompose of the $\mathrm{X}$. Hence, the register at the end of each adder does not always store the final result of such operations. It may store the partial sum instead. A multiplexer (MUX) would select either the partial results or the bias. Lasers inject light based on the selection.

(a) A 5x5x1 input feature as the layer input

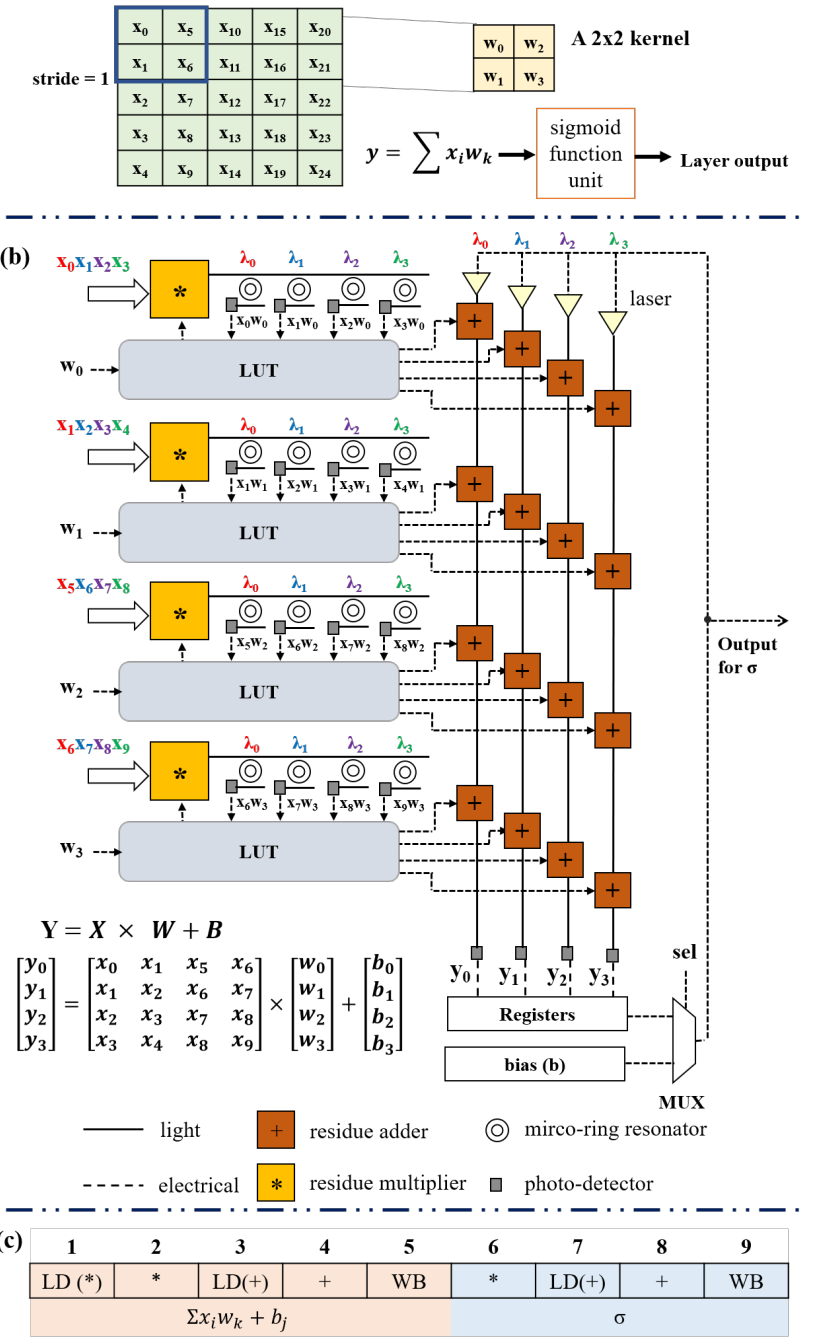

Figure 5: A Matrix-Vector Multiplication with Example of Integrated Photonic R-MVM Unit when WDM=4 and Its Corresponding Pipeline. (a) An example of a convolutional neural network with a $5 \times 5$ input feature map and a $2 \times 2$ kernel. (b) Mapping the example from (a) to an R-MVM (WDM=4). (c) A pipeline with one operation in a convolutional layer that comes with a sigmoid activation function.

In general, the overall time that is required for several modulo-m MAC operations is shown in equation (1).

$$
\begin{aligned}
& T_{R-M V M}=\quad T_{\text {init }}+T_{o p t_{-} m u l}+T_{O E} \\
& +T_{\text {init }}+w * T_{\text {opt_add }}+T_{O E}
\end{aligned}
$$

where $T_{R-M V M}$ represents the overall time requirement for all MAC operations performed in the one proposed R-MVM unit; $T_{\text {init }}$ represents the initialization time for the nanophotonic residue blocks, including loading the switch states from LUT, setting up the switches, and generating lights from lasers; $T_{o p t \_m u l}$ and $T_{o p t} a d d$ represent the propagation time of the light passing through the residue multipliers and residue adders, respectively; $T_{O E}$ stands for the time of which an optical signal is converted to electrical signal, which is the response time for the ring resonator and photodetector; $w$ represents the number of wavelengths.

\subsection{Sigmoid Activation Function Units}

Typically, once representing neurons is done the MAC calculations, activation functions would be applied to the results. The sigmoid function is popular as activation functions in deep neural networks and is designed in proposed work. However, floating point calculations exist in such function. Hence, Taylor series, expressed as a polynomial, is selected to express the sigmoid function, which contains residue adders and multipliers only.

Consider the polynomial $P(x)=a x^{4}+b x^{3}+c x^{2}+d x+e$. Tai et al. further utilized the residue adders and multipliers [55]. A mapping for the terms that include $\mathrm{x}$, e.g. $x^{4}, x^{3}$ and so on, would be implemented at first. Then the coefficients would be applied as the selection signal for residue multipliers. Several adders would be applied to accumulate all the products that are derived from the mapped multipliers. The schematic is shown in Figure 6. It allows the whole system to be programmable to any coefficient. The tradeoff of this architecture is that the specific design for each power of a particular modulus should be implemented in advance. It reduces the flexibility of the system and increases the complexity of the design.

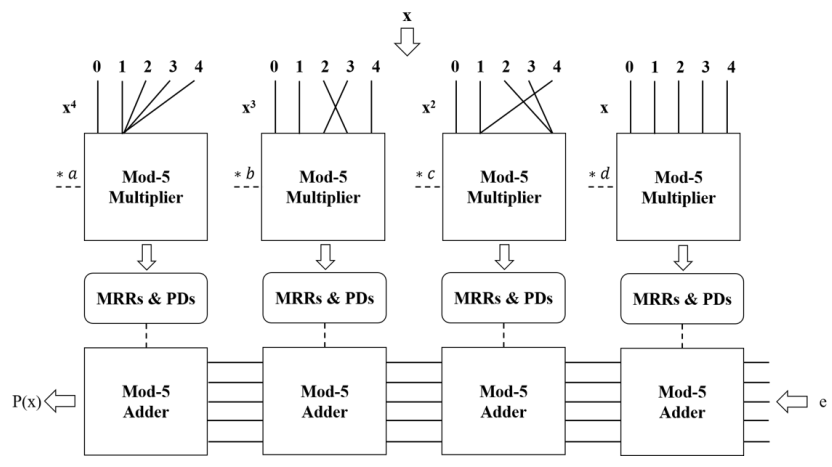

Figure 6: A Modulo-5 Polynomial Evaluation with Residue Multipliers and Adders.

For each polynomial, all multiplications could be run in parallel and thus the overall delay in both the sigmoid function and the hyperbolic tangent function with $n$ elements in Taylor series would be

$$
\begin{aligned}
T_{\text {poly }}= & T_{\text {init }}+T_{\text {opt_mul }}+T_{O E} \\
& +T_{\text {init }}+n * T_{\text {opt_add }}+T_{O E}
\end{aligned}
$$

where the $T_{\text {poly }}$ represents the time for a single polynomial calculation, $n$ stands for the number of terms used in Taylor series, 
and other terms are the same as described in equation (1). However, the hardware requires more attention in design time since each term for the power of $x$ should be processed in advance and specific hardware would be fabricated accordingly. In addition, if the activation function is always the same type, then the switch requires one-time initialization only because the coefficient in the Taylor series for one specific function is always the same.

\subsection{Convolutional Intra-Layer Pipeline Design}

The operations within a convolutional neural layer, including the MAC operation as well as the activation function, could be pipelined, as shown in Figure 5 (c). Assuming that the time of reading data from the LUT is one cycle, which is around $0.5 \mathrm{~ns}$ (simulation result from CACTI 7.0 [34]). After loading the switches states, the residue adder/multiplier would be set to route the light properly. All the switch settings could be done concurrently, and the response time of a photonic switch could be as low as 5.1 ps [52]. Then light could be injected and traverses at extremely high speed, and it costs less than 1ps for a residue computing engine propagation at the worst case. An MRR resonates light at around $130 \mathrm{GHz}$, and a PD detects light at around 35 110 GHz. By setting the lower bound of the $35 \mathrm{GHz}$, both the MRR and PD require around 28ps as the response time. Thus, the overall light traveling time, from injection to detection, would be around 60ps, which is much shorter than the designed clock cycle. To guarantee that the optical switches are being set before light injected, a single cycle could be separated as two sub-cycles. The switch will be set up in the first half cycle, and the light will be injected and detected in the second half cycle.

All the arithmetic operations are executed in optical residue computing engines and consume one cycle (cycle 2 and cycle 4). Loading the corresponding data and the states of the switches should be done before setting them up, which costs one cycle (cycle 1 and cycle 3 ). In the end, results should be stored in the registers, consuming one cycle (cycle 5). However, taking advantage of the highly parallel architecture, several MAC operations could be done in five cycles simultaneously. The bias addition is included as well. The sigmoid activation function requires four cycles through the Taylor series could be considered as a MAC operation. Since the coefficients of a Taylor series of sigmoid functions are constant, the switches' states of multipliers in the polynomial will not change. Therefore, it could be pre-loaded at the design time, resulting in the saving of one cycle. Thus, the computations inside a convolutional neuron, including the dot-product operations with bias addition, as well as the sigmoid activation function, could be obtained at the end of cycle 9. Currently, only the intra-layer pipeline of the convolutional layer is considered in this work. Inter-layer pipelining, including scheduling the neural network layers and hardware resources, requires much effort is the focus of our future work.

\subsection{Max Pooling Unit}

A residue max pooling unit is designed to reduce the conversions between the binary and the residue domain. In order to find the maximum value, first, a subtractor is needed, which could be easily developed by the residue adder since negative notation is available in residue arithmetic. Then a sign detector would be useful to identify the larger number according to the result of the subtraction.
The sign in the RNS is implicit, which means the sign is part of the number representation itself. Thus, it is not straightforward to determine if a residue representation is positive or negative. In conventional number systems, the sign representation is explicit. The two's complement representation of the binary system, for example, utilizes the most significant bit to represent the sign itself. It is straightforward to do either sign detection or comparison in the weighted system, hence converting a residue number to a weighted system is proposed to detect the sign with the Chinese Remainder Theorem [51, 53]. However, reverting the residue representation to a conventional number system would waste resources and lose the advantage of residue computing. Thus, researchers proposed a mixed-radix conversion process for residue sign detection, which involves subtraction and multiplication [18, 39, 53].

A mixed-radix number system represents a number with different radices for each digit. In such a number system, for selected radices, $\left\{R_{n}, R_{n-1}, \ldots, R_{1}\right\}$, each digit would have its own weights, $\left\{a_{n}, a_{n-1}, \ldots, a_{1}\right\}\left(0 \leq a_{i}<R_{i}\right)$. Then a number $X$ could use the following equation to obtain the natural number format.

$$
X=a_{n} \prod_{i=1}^{N-1} R_{i}+\ldots+a_{3} R_{1} R_{2}+a_{2} R_{1}+a_{1}
$$

Assuming that the moduli set are the same as the radices, the only problem left is to determine the coefficients of $\left\{a_{n}, a_{n-1}, \ldots, a_{1}\right\}$, when converting the residue number system to the mixed-radix number system. $a_{1}$ always equals to $|x|_{R_{1}}$ because $a_{1}$ is not multiplied by $R_{1}$. From equation (3), $a_{2}=\left|\frac{X-a_{1}}{R_{1}}\right|_{R_{1}}$. It is interesting to note that all the moduli should be relatively prime to each other. Therefore, the multiplicative inverse, denoted by $\left|x^{-1}\right|_{m}$, of different moduli, $m$, can be computed using a division procedure in this case. By successive subtractions of $a_{i-1}$ and divisions of the multiplicative inverse for each modulus, the weights $a_{i}$ of each digit of such a mixed-radix number system can be obtained.

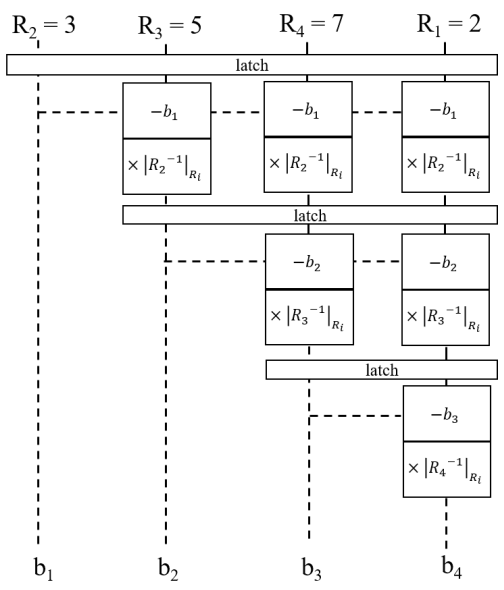

Figure 7: Sign Detection in Residue Domain by Converting to Weighted Number System.

As shown in Figure 7, by aligning the moduli in a different order, and following the procedure of subtraction and multiplicative 
inverse multiplication, the sign of residue could be detected using the last two digits. First, the moduli are aligned in ascending order, and then the even modulus 2 is placed at the end. For instance, if the moduli set is $\{2,3,5,7\}$, then now the new order should be $\{3,5$, $7,2\}$. A truth table stores all the possible combinations, and then the sign could be derived.

For a 16-bit binary system, we selected seven smallest primes as the moduli set. It requires 12 cycles for sign detection only. Two more cycles are applied additionally for the subtraction, which consumes a relatively long time. The good thing is that this design is easy to be pipelined with the latches shown in Figure 7 since all the computations are irrelevant to each other in each clock cycle. Therefore, an ideal pipeline could be designed, e.g., one operation would be completed per cycle. However, extra hardware is needed in addition to latches, including additional lasers, MRRs and PDs. Note that this is for two-number comparison only. In most of the current CNNs, the kernel of a max pooling layer would be $2 \times 2$. Therefore, we utilized three max pooling units to perform the comparison in DNNARA design.

\section{EVALUATION}

\subsection{Experimental Setup}

The hardware parameters are specified in Table 1. CACTI 7.0 [34] is utilized to model the area and energy consumption for LUTs, registers and buses. Lumerical is used to simulate the optical building blocks. Moreover, we built on top of those tools, our own systemlevel simulator, to evaluate the overall performance of DNNARA. We manually mapped each of the benchmarks to the R-MVMs, tiles and chips, and measured the delay and power consumption.

The proposed R-MVM contains one set of $2 \times 2$ switches, lasers, MRRs, and PDs. The power consumption is varied with the switch states, which is corresponding to the actual input. Here we assume that there is a $50 \%$ probability that the switch would be in bar state. The $2 \times 2$ switches are adapted from the [52]. Since the off-chip lasers require additional splitters to inject light into the corresponding position, on-chip lasers are chosen in our design. Although the total amount of lasers increases, they will not work at the same time. We considered the worst case; that is, no light will be injected in the same port. The number of operating lasers would be the same as the WDM size of each multiplier. Lasers' parameters are adapted from [40]. MRRs are designed for specific wavelength only, which means it acts like the waveguide. Hence, it consumes no dynamic energy in this circumstance. Parameters of MRRs and PDs are adapted from [57] and [46], respectively. HyperTransport serial link model of DaDianNao [12] is utlized in this analysis.

To evaluate the whole system, we introduce the metric of the computational capability, which is represented as the number of 16bit operations per second per $\mathrm{mm}^{2}$ per Watt $\left(G O P s /\left(s \cdot \mathrm{mm}^{2} \cdot W\right)\right.$ ). This metric helps us to evaluate the whole system in a more general way, considering the computing throughput, area, and energy at the same time. In addition, we apply our architecture on modern neural network architectures for further performance evaluation. We adapted the VGGs and DeepFace from ISAAC [47], ResNets [24], as well as modified LeNet-5 [29]. The configurations of testing benchmarks are shown in Table 2 . The activation function at the end of each layer is not shown. Noted that we evaluated the benchmarks
Table 1: DNNARA Hardware Parameters

\begin{tabular}{|c|c|c|c|c|}
\hline \multicolumn{5}{|c|}{ DNNARA Design at $1.2 \mathrm{GHz}(32 \mathrm{~nm})$} \\
\hline Component & Params & Spec. & $\operatorname{Area}\left(\mathrm{mm}^{2}\right)$ & Power(mW) \\
\hline Memory & $\begin{array}{c}\text { size } \\
\text { bus_width }\end{array}$ & $\begin{array}{c}256 \mathrm{~KB} \\
64 \mathrm{~b}\end{array}$ & 0.29 & 28.2 \\
\hline bus & num_wire & 384 & 0.09 & 7 \\
\hline Router & flit_size & 32 & $\begin{array}{c}0.151 \\
\text { (shared by } \\
4 \text { tiles) }\end{array}$ & 42 \\
\hline Sigmoid & number & 2 & 0.053 & 24.6 \\
\hline MaxPool & number & 1 & 0.026 & 11.3 \\
\hline R-Accu & number & 1 & 0.005 & 3.63 \\
\hline \multicolumn{5}{|c|}{ PR-MVM Characteristics } \\
\hline R-multiplier & $\begin{array}{c}\text { number } \\
\text { WDM }\end{array}$ & $\begin{array}{l}5 \\
5\end{array}$ & 0.018 & 7.23 \\
\hline R-adder & number & 25 & 0.075 & 29.48 \\
\hline Laser & number & 530 & 0.0053 & 0.13 \\
\hline MRR\&PD & number & 530 & 0.014 & 10.6 \\
\hline Total & & & 0.112 & 47.43 \\
\hline \multicolumn{5}{|c|}{ Chip Configuration } \\
\hline $\begin{array}{l}1 \text { Tile } \\
1 \text { Chip }\end{array}$ & $\begin{array}{c}\text { R-MVM } \\
\text { num_Tile }\end{array}$ & $\begin{array}{c}32 \\
8\end{array}$ & $\begin{array}{c}4.09 \\
32.76\end{array}$ & $\begin{array}{l}1.62 \mathrm{~K} \\
13.0 \mathrm{~K}\end{array}$ \\
\hline Hyper Tr & $\begin{array}{c}\text { num_link } \\
\text { link freq } \\
\text { link bw }\end{array}$ & $\begin{array}{c}4 \\
1.6 \mathrm{GHz} \\
6.4 \mathrm{~GB} / \mathrm{s}\end{array}$ & 22.88 & $10.4 \mathrm{~K}$ \\
\hline Chip Total & & & 55.64 & $23.4 \mathrm{~K}$ \\
\hline
\end{tabular}

Table 2: Configuration of Benchmarks

\begin{tabular}{|c|c|c|c|}
\hline Name & \# of Layers & Input Size & \# of Weights \\
\hline \hline LeNet-5 & 5 & 32 & $61 \mathrm{~K}$ \\
\hline VGG-1 & 11 & 224 & $133 \mathrm{M}$ \\
VGG-2 & 16 & 224 & $134 \mathrm{M}$ \\
VGG-3 & 16 & 224 & $138 \mathrm{M}$ \\
VGG-4 & 19 & 224 & $144 \mathrm{M}$ \\
\hline ResNet-18 & 18 & 224 & $36 \mathrm{M}$ \\
ResNet-34 & 34 & 224 & $46 \mathrm{M}$ \\
ResNet-50 & 50 & 224 & $12 \mathrm{M}$ \\
\hline DeepFace & 7 & 152 & $46 \mathrm{M}$ \\
\hline
\end{tabular}

as the natural way, which means a single R-MVM unit would be utilized to generate a value of one layer. Thus, the results would be even better if there is a smarter compiler, which will be the future work.

- Design Space Exploration. The computational capability is affected by several parameters, including the WDM size, the number of R-MVM in a tile and the number of tiles per chip. To derive the maximum computational capability of the system, we tested the above parameters at the peak performance, when all computational units are working concurrently. Figure 8 shows that the optimal computational capability is $12.6 \mathrm{GOPs} /\left(\mathrm{s} \cdot \mathrm{mm}^{2}\right.$. $W$ ), with 5 WDM, 32 R-MVMs in a tile, and 8 tiles in a chip as the configuration. The computational capability reaches the peak for a specific number of R-MVMs in a tile for different WDM sizes and different numbers of tiles on a chip. The computational capability does not always increase as we enlarge the number of R-MVMs that is being utilized. This is because the more R-MVMs on the chip, the more power is required. As the number of tiles and/or the number of R-MVMs increases, both the area and power increase. However, the computing throughput does not increase proportionally since the area and power overhead are varied with different coefficients. Thus, we gain no benefit after reaching a certain point. In general, the more WDM, the higher computational capability is achieved. 
However, the increasing proportion does not match the increment of WDM size since the area and power overhead are varied with different coefficients.

\subsection{Results}

- Peak Throughput. Utilizing our optimal configuration for the proposed DNNARA architecture, the peak computational throughput of one R-MVM would be 30 GOPs/s. However, the computational throughput of the ISAAC [47] design reaches $0.34 \mathrm{GOPs} / \mathrm{s}$ for one memristor crossbar array. The peak throughput of a single unit that executes MAC operation is two orders of magnitude higher than the one of ISAAC. The overall throughput of the system will be less than the peak performance of our optical R-MVM as we add slower components to the system (This will be discussed in the evaluation of benchmarks). Moreover, optical components, in general, are large, and thus our design requires more area. Hence, the computation throughput per unit area is smaller than the one of ISAAC. Nevertheless, the high speed of the photonic component guarantees high computation throughput. Therefore, fewer units are required in our design to reach the same performance. The proposed DNNARA chip requires 8 tiles, and the area and power budget are $55.64 \mathrm{~mm}^{2}$ and 23.4 Watt, respectively. On the other hand, the ISSAC design requires 168 tiles on a chip, and the area and power consumption are $88 \mathrm{~mm}^{2}$ and 65.81 Watt. DNNARA design uses less number of units, less area, and less power to achieve higher performance.

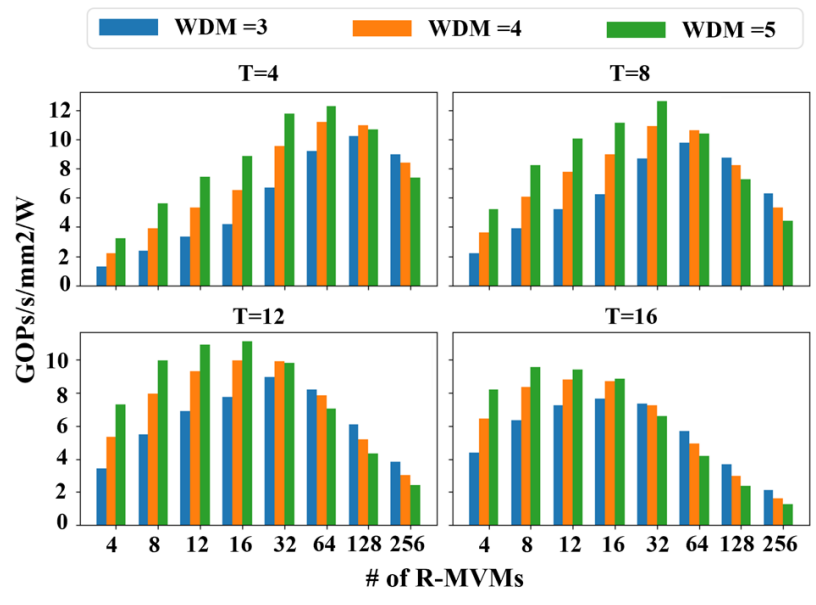

Figure 8: Computational Capability with Different Configurations of DNNARA Design. T represents the number of tiles on a chip.

- Accuracy. The accuracy of a neural network is one of the most important properties. There are two possible sources of accuracy loss when it comes to photonic systems. The first one is due to the use of lower precision. Previous works [12, 20, 47] proved that using 16-bit precision is sufficient in such machine learning algorithms. Although the same data width has been chosen in the proposed work, the RNS domain requires the module transform between binary and residue domain. In addition, Taylor Expansion

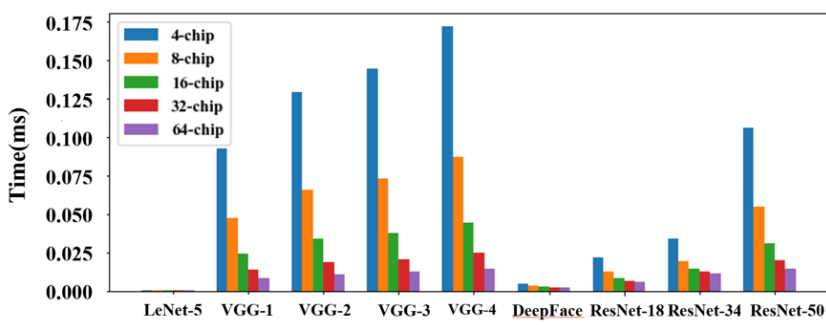

(a)

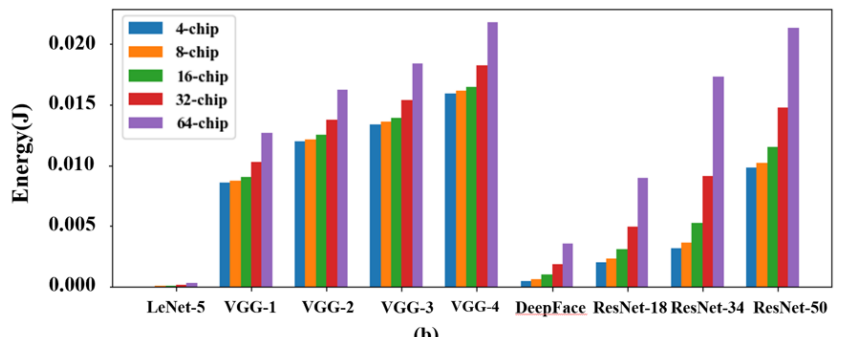

(b)

Figure 9: (a) Delay and (b) Energy Consumption of DNNARA to Run CNN Benchmarks.

might cause inaccuracy here. However, RNSnet [45] has studied the accuracy of DNN in RNS. A 16-bit DNN (our work) could reach 0\% quality loss compared to the baseline of different types. Moreover, they proposed utilizing Taylor's expansion for activation function to avoid multiple conversion between binary system and RNS. Thus the proposed DNNARA architecture should not lose accuracy with 16-bit data width compared to other 16-bit methods. The other source of inaccuracy is due to the conversion from analog optical signals to digital electrical signals. However, in our system, we use one-hot-encoding in the optical domain, which relieves the requirement of using the analog to digital conversions and results in no accuracy loss. Thus, our implementation provides the same accuracy as the baseline. The third resource comes from the multiwavelength propagation in a single fiber. Protocol ITU-T G.694.1 [59] recommends that the frequency spacing for a dense wavelength division multiplexing (DWDM) system is around $0.8 \mathrm{~nm}$. It could fit up to more than 130 wavelengths using the selected switches [52]. Practically to avoid the effect of crosstalk and power consumption, we restrict ourselves to $3 \sim 5$ wavelengths. However, due to the tradeoff between the benefits of WDM and the extra overhead due to the additional filters, including sets of modulators and photo-detectors, a set of 5 wavelengths is selected.

- CNN Benchmark Evaluation. Figure 9 depicts the running time and energy consumption for the tested benchmarks. Both the time and energy consumption are related to the benchmark since the benchmark size varies from each other. Compared to VGG, LeNet-5 and DeepFace are relatively small as shown in Table 2. Thus, these two benchmarks cost a shorter time and less energy for the operation than the VGG. In general, the more chips utilized, the shorter time required. However, it does not proportionally decrease as we increase the number of chips due to the communication overhead between chips. Similarly, energy consumption grows as the number of utilized chips increases. Communication costs much energy though the operating time reduces. 
Most of the time, the chip is working for the MAC operations in convolutional layers, if VGG-4 is running. As the kernel size decreases, the time drops as well. Although a fully connected layer contains a large number of weights, they are independent of each other, which could be mapped to different R-MVM units. Pooling does not consume too much time, although there is one pooling unit per tile, because the max pooling layer could be pipelined. Thus, the computational efficiency of such a unit is high, as well as the throughput. NVIDIA reports that one of the most recent GPUs, Tesla V100, could perform one image in $1.2 \mathrm{~ms}$ when running VGG4 [16] while the power consumption is 250 Watt [17]. The batch size equals to one for inference in this scenario. With a similar power budget, 12 DNNARA chips could be used, and one image could be processed in $0.062 \mathrm{~ms}$ in such a system, achieving a speedup of 19.3x.

\subsection{Comparison with other NN Accelerators}

To better address our contribution, we conclude and compare proposed work to i) electrical NN accelerators based on RNS, and ii) optical NN accelerators without RNS.

- Electrical RNS NN Accelerators. Work NEUROM [4], RNSTPU [38], and NRNS [35] are built based on FPGA. With the absence of the data of area and power, we could only compare with the throughput. DNNARA reaches 12.6 TOPS/s (tera-operations/second), while the others reach $500 \mathrm{MOPS} / \mathrm{s}, 23.25 \mathrm{GOPS} / \mathrm{s}$ and $132.2 \mathrm{GOPS} / \mathrm{s}$, respectively. The major limitation of such technology is due to the LUT. Traditionally, electrical RNS implementation utilized LUT to store the results; alternatively, DNNARA stores the switch states only. With the smaller size of memory, DNNARA loads data in a shorter time and consumes both less area and power.

- Optical NN Accelerators. Recent works which implemented the optical $\mathrm{NN}$ accelerators with different technologies have been proposed, including microdisk weight banks [33], microring weight banks [56], diffractive optical layers [31], and Mach-Zehnder interferometers [48]. HolyLight [33] proposed the optical accelerator as a chip-size system-level design, e.g., considering the hardware support, including memory and interconnect. Two architectures were proposed. The first implementation requires the use of ADCs and DACs, whereas the second implementation eliminates the need for ADCs but uses less accurate quantized CNN. HolyLight-M performs slightly better than proposed DNNARA. However, a higher frequency has been deployed on their system, which is ten times faster $(12.8 \mathrm{GHz})$ than ours. DNNARA architecture, due to the conservative design, is running at the speed of $1.2 \mathrm{GHz}$ only. If we run our system at the same speed, we are $7 \mathrm{x}$ faster than HolyLight.

\section{CONCLUSION}

In this paper, we introduce a novel CNN accelerator, DNNARA, that is based on residue arithmetic and integrated photonics. The use of RNS enables us to reduce the optical critical path in order to maintain low laser power. Moreover, the one-hot encoding, used to encode the numbers, enhances the speed of switching between the optical and electronic domain, enabling our system to have high throughput. We take advantage of WDM to achieve a high level of parallelism while maintaining a reasonable area. Our residue MVM module can achieve two orders of magnitude higher throughput when compared to the memristor crossbar. When using the same power budget as the NVIDIA Tesla V100 GPU, our chips can do inference more than 19.3 times faster.

\section{ACKNOWLEDGMENTS}

This project is supported by Air Force Office of Scientific Research (AFOSR) award number FA9550-19-1-0277.

\section{REFERENCES}

[1] Hossam O Ahmed, Maged Ghoneima, and Mohamed Dessouky. 2018. Concurrent MAC unit design using VHDL for deep learning networks on FPGA. In 2018 IEEE Symposium on Computer Applications \& Industrial Electronics (ISCAIE). IEEE, 31-36.

[2] Hossam O Ahmed, Maged Ghoneima, and Mohamed Dessouky. 2018. High-Speed 2D Parallel MAC Unit Hardware Accelerator for Convolutional Neural Network. In Proceedings of SAI Intelligent Systems Conference. Springer, 655-663.

[3] Ahmad Qaisar Ahmad Al Badawi, Yuriy Polyakov, Khin Mi Mi Aung, Bharadwaj Veeravalli, and Kurt Rohloff. 2019. Implementation and performance evaluation of RNS variants of the BFV homomorphic encryption scheme. IEEE Transactions on Emerging Topics in Computing (2019).

[4] Giuseppe Alia and Enrico Martinelli. 2005. NEUROM: a ROM based RNS digital neuron. Neural networks 18, 2 (2005), 179-189.

[5] Md Zahangir Alom, Tarek M Taha, Christopher Yakopcic, Stefan Westberg, Paheding Sidike, Mst Shamima Nasrin, Brian C Van Esesn, Abdul A S Awwal, and Vijayan K Asari. 2018. The history began from alexnet: A comprehensive survey on deep learning approaches. arXiv preprint arXiv:1803.01164 (2018).

[6] Aayush Ankit, Izzat El Hajj, Sai Rahul Chalamalasetti, Geoffrey Ndu, Martin Foltin, R Stanley Williams, Paolo Faraboschi, Wen-mei W Hwu, John Paul Strachan, Kaushik Roy, et al. 2019. PUMA: A programmable ultra-efficient memristorbased accelerator for machine learning inference. In Proceedings of the TwentyFourth International Conference on Architectural Support for Programming Languages and Operating Systems. ACM, 715-731.

[7] Leily A Bakhtiar and Mehdi Hosseinzadeh. 2016. All optical residue arithmetic with micro ring resonators and its application. Optical and Quantum Electronics 48, 2 (2016), 125.

[8] Leily A Bakhtiar, Elham Yaghoubi, Seyedeh Mehri Hamidi, and Mehdi Hosseinzadeh. 2015. Optical RNS adder and multiplier. International fournal of Computer Applications in Technology 52, 1 (2015), 71-76.

[9] Václav E Beneš. 1964. Permutation groups, complexes, and rearrangeable connecting networks. Bell System Technical fournal 43, 4 (1964), 1619-1640.

[10] Chihming Chang and Rami Melhem. 1997. Arbitrary size benes networks. Parallel Processing Letters 7, 03 (1997), 279-284.

[11] Tianshi Chen, Zidong Du, Ninghui Sun, Jia Wang, Chengyong Wu, Yunji Chen, and Olivier Temam. 2014. Diannao: A small-footprint high-throughput accelerator for ubiquitous machine-learning. In ACM Sigplan Notices, Vol. 49. ACM, 269-284.

[12] Yunji Chen, Tao Luo, Shaoli Liu, Shijin Zhang, Liqiang He, Jia Wang, Ling Li, Tianshi Chen, Zhiwei Xu, Ninghui Sun, et al. 2014. Dadiannao: A machinelearning supercomputer. In Proceedings of the 47th Annual IEEE/ACM International Symposium on Microarchitecture. IEEE Computer Society, 609-622.

[13] Ping Chi, Shuangchen Li, Cong Xu, Tao Zhang, Jishen Zhao, Yongpan Liu, Yu Wang, and Yuan Xie. 2016. Prime: A novel processing-in-memory architecture for neural network computation in reram-based main memory. In ACM SIGARCH Computer Architecture News, Vol. 44. IEEE Press, 27-39.

[14] Dan Claudiu Ciresan, Ueli Meier, Jonathan Masci, Luca Maria Gambardella, and Jürgen Schmidhuber. 2011. Flexible, high performance convolutional neural networks for image classification. In Twenty-Second International foint Conference on Artificial Intelligence.

[15] Jason Cong and Bingjun Xiao. 2014. Minimizing computation in convolutional neural networks. In International conference on artificial neural networks. Springer, 281-290.

[16] NVIDIA Corporation. 2019. NVIDIA Tesla Deep Learning Product Performance. https://developer.nvidia.com/deep-learning-performance-training-inference

[17] NVIDIA Corporation. 2019. NVIDIA TESLA V100 TENSOR CORE GPU. https: //www.nvidia.com/en-us/data-center/tesla-v100/

[18] Giovanni Dimauro, Sebastiano Impedovo, and Giuseppe Pirlo. 1993. A new technique for fast number comparison in the residue number system. IEEE transactions on computers 42, 5 (1993), 608-612.

[19] Kaiyuan Guo, Shulin Zeng, Jincheng Yu, Yu Wang, and Huazhong Yang. 2017. A survey of fpga-based neural network accelerator. arXiv preprint arXiv:1712.08934 (2017).

[20] Suyog Gupta, Ankur Agrawal, Kailash Gopalakrishnan, and Pritish Narayanan. 2015. Deep learning with limited numerical precision. In International Conference on Machine Learning. 1737-1746. 
[21] Alexander Guzhva, Sergey Dolenko, and Igor Persiantsev. 2009. Multifold acceleration of neural network computations using GPU. In International Conference on Artificial Neural Networks. Springer, 373-380.

[22] Shai Halevi, Yuriy Polyakov, and Victor Shoup. 2019. An improved RNS variant of the BFV homomorphic encryption scheme. In Cryptographers' Track at the RSA Conference. Springer, 83-105.

[23] Ryan Hamerly, Liane Bernstein, Alexander Sludds, Marin Soljačić, and Dirk Englund. 2019. Large-scale optical neural networks based on photoelectric multiplication. Physical Review X 9, 2 (2019), 021032.

[24] Kaiming He, Xiangyu Zhang, Shaoqing Ren, and Jian Sun. 2016. Deep residual learning for image recognition. In Proceedings of the IEEE conference on computer vision and pattern recognition. $770-778$.

[25] Alan Huang, Yoshito Tsunoda, Joseph W Goodman, and Satoshi Ishihara. 1979 Optical computation using residue arithmetic. Applied Optics 18, 2 (1979), 149162 .

[26] Mohsen Imani, Saransh Gupta, Yeseong Kim, and Tajana Rosing. 2019. Floatpim: In-memory acceleration of deep neural network training with high precision. In Proceedings of the 46th International Symposium on Computer Architecture. ACM, 802-815.

[27] Norman P Jouppi, Cliff Young, Nishant Patil, David Patterson, Gaurav Agrawal, Raminder Bajwa, Sarah Bates, Suresh Bhatia, Nan Boden, Al Borchers, et al 2017. In-datacenter performance analysis of a tensor processing unit. In 2017 ACM/IEEE 44th Annual International Symposium on Computer Architecture (ISCA) IEEE, $1-12$

[28] Abbas Karimi, Kiarash Aghakhani, Seyed Ehsan Manavi, Faraneh Zarafshan, and SAR Al-Haddad. 2014. Introduction and analysis of optimal routing algorithm in benes networks. Procedia Computer Science 42 (2014), 313-319.

[29] Yann LeCun, Léon Bottou, Yoshua Bengio, Patrick Haffner, et al. 1998. Gradientbased learning applied to document recognition. Proc. IEEE 86, 11 (1998), 22782324.

[30] Huimin Li, Xitian Fan, Li Jiao, Wei Cao, Xuegong Zhou, and Lingli Wang. 2016 A high performance FPGA-based accelerator for large-scale convolutional neural networks. In 2016 26th International Conference on Field Programmable Logic and Applications (FPL). IEEE, 1-9.

[31] Xing Lin, Yair Rivenson, Nezih T Yardimci, Muhammed Veli, Yi Luo, Mona Jarrahi, and Aydogan Ozcan. 2018. All-optical machine learning using diffractive deep neural networks. Science 361, 6406 (2018), 1004-1008.

[32] Daofu Liu, Tianshi Chen, Shaoli Liu, Jinhong Zhou, Shengyuan Zhou, Olivier Teman, Xiaobing Feng, Xuehai Zhou, and Yunji Chen. 2015. Pudiannao: A polyvalent machine learning accelerator. In ACM SIGARCH Computer Architecture News, Vol. 43. ACM, 369-381.

[33] Weichen Liu, Wenyang Liu, Yichen Ye, Qian Lou, Yiyuan Xie, and Lei Jiang. 2019. HolyLight: A Nanophotonic Accelerator for Deep Learning in Data Centers. In 2019 Design, Automation \& Test in Europe Conference \& Exhibition (DATE). IEEE, 1483-1488.

[34] Naveen Muralimanohar, Rajeev Balasubramonian, and Norm Jouppi. 2007. Optimizing NUCA organizations and wiring alternatives for large caches with CACTI 6.0. In Proceedings of the 40th Annual IEEE/ACM International Symposium on Microarchitecture. IEEE Computer Society, 3-14.

[35] Hiroki Nakahara and Tsutomu Sasao. 2015. A deep convolutional neural network based on nested residue number system. In 2015 25th International Conference on Field Programmable Logic and Applications (FPL). IEEE, 1-6.

[36] Dong Nguyen, Daewoo Kim, and Jongeun Lee. 2017. Double MAC: Doubling the performance of convolutional neural networks on modern FPGAs. In Design Automation \& Test in Europe Conference \& Exhibition (DATE), 2017. IEEE, 890-893.

[37] Yuanhui Ni, Keni Qiu, Weiwen Chen, Lixue Xia, and Yu Wang. 2018. Low power driven loop tiling for RRAM crossbar-based CNN. In Proceedings of the 33rd Annual ACM Symposium on Applied Computing. ACM, 375-380.

[38] Eric B Olsen. 2018. RNS Hardware Matrix Multiplier for High Precision Neural Network Acceleration:" RNS TPU". In 2018 IEEE International Symposium on Circuits and Systems (ISCAS). IEEE, 1-5.

[39] Amos R Omondi and Benjamin Premkumar. 2007. Residue number systems: theory and implementation. Vol. 2. World Scientific.

[40] Rupert F Oulton, Volker J Sorger, Thomas Zentgraf, Ren-Min Ma, Christopher Gladden, Lun Dai, Guy Bartal, and Xiang Zhang. 2009. Plasmon lasers at deep subwavelength scale. Nature 461, 7264 (2009), 629.

[41] Jiaxin Peng, Yousra Alkabani, Shuai Sun, Volker Sorger, and Tarek El-Ghazawi. 2019. Integrated Photonics Architectures for Residue Number System Computations. In IEEE International Conference on Rebooting Computing (ICRC 2019). 129-137.

[42] Jiaxin Peng, Shuai Sun, Vikram K Narayana, Volker J Sorger, and Tarek ElGhazawi. 2018. Residue number system arithmetic based on integrated nanophotonics. Optics letters 43, 9 (2018), 2026-2029.

[43] S Preethi, V Prasannadevi, and B Arunadevi. 2020. Smart Healthcare Monitoring System for War-End Soldiers Using CNN. In Smart Medical Data Sensing and IoT Systems Design in Healthcare. IGI Global, 97-131.

[44] Vinyas D Sagar and TS Nanjundeswaraswamy. 2019. Artificial Intelligence in Autonomous Vehicles-a Literature Review. i-Manager's fournal on Future
Engineering and Technology 14, 3 (2019), 56.

[45] Sahand Salamat, Mohsen Imani, Sarangh Gupta, and Tajana Rosing. 2018. Rnsnet: In-memory neural network acceleration using residue number system. In 2018 IEEE International Conference on Rebooting Computing (ICRC). IEEE, 1-12.

[46] Yannick Salamin, Ping Ma, Benedikt Baeuerle, Alexandros Emboras, Yuriy Fedoryshyn, Wolfgang Heni, Bojun Cheng, Arne Josten, and Juerg Leuthold. 2018. $100 \mathrm{GHz}$ plasmonic photodetector. ACS photonics 5, 8 (2018), 3291-3297.

[47] Ali Shafiee, Anirban Nag, Naveen Muralimanohar, Rajeev Balasubramonian, John Paul Strachan, Miao Hu, R Stanley Williams, and Vivek Srikumar. 2016. ISAAC: A convolutional neural network accelerator with in-situ analog arithmetic in crossbars. ACM SIGARCH Computer Architecture News 44, 3 (2016), 14-26.

[48] Yichen Shen, Nicholas C Harris, Scott Skirlo, Mihika Prabhu, Tom Baehr-Jones, Michael Hochberg, Xin Sun, Shijie Zhao, Hugo Larochelle, Dirk Englund, et al. 2017. Deep learning with coherent nanophotonic circuits. Nature Photonics 11, 7 (2017), 441.

[49] Karen Simonyan and Andrew Zisserman. 2014. Very deep convolutional networks for large-scale image recognition. arXiv preprint arXiv:1409.1556 (2014).

[50] Linghao Song, Xuehai Oian, Hai Li, and Yiran Chen. 2017. Pipelayer: A pipelined reram-based accelerator for deep learning. In 2017 IEEE International Symposium on High Performance Computer Architecture (HPCA). IEEE, 541-552.

[51] Leonel Sousa and Paulo Martins. 2017. Sign Detection and Number Comparison on RNS 3-Moduli Sets $\left\{2^{\wedge} \mathrm{n}-1,2^{\wedge}\{\mathrm{n}+\mathrm{x}\}, 2^{\wedge} \mathrm{n}+1 \backslash\right\}$. Circuits, Systems, and Signal Processing 36, 3 (2017), 1224-1246.

[52] Shuai Sun, Vikram K Narayana, Ibrahim Sarpkaya, Joseph Crandall, Richard A Soref, Hamed Dalir, Tarek El-Ghazawi, and Volker J Sorger. 2017. Hybrid photonicplasmonic nonblocking broadband $5 \times 5$ router for optical networks. IEEE Photonics fournal 10, 2 (2017), 1-12.

[53] Nicholas S Szabo and Richard I Tanaka. 1967. Residue arithmetic and its applications to computer technology. McGraw-Hill.

[54] Christian Szegedy, Wei Liu, Yangqing Jia, Pierre Sermanet, Scott Reed, Dragomir Anguelov, Dumitru Erhan, Vincent Vanhoucke, and Andrew Rabinovich. 2015. Going deeper with convolutions. In Proceedings of the IEEE conference on computer vision and pattern recognition. $1-9$.

[55] A Tai, I Cindrich, James R Fienup, and CC Aleksoff. 1979. Optical residue arithmetic computer with programmable computation modules. Applied optics 18, 16 (1979), 2812-2823.

[56] Alexander N Tait, Thomas Ferreira De Lima, Ellen Zhou, Allie X Wu, Mitchell A Nahmias, Bhavin J Shastri, and Paul R Prucnal. 2017. Neuromorphic photonic networks using silicon photonic weight banks. Scientific reports 7, 1 (2017), 1-10.

[57] Erman Timurdogan, Cheryl M Sorace-Agaskar, Jie Sun, Ehsan Shah Hosseini, Aleksandr Biberman, and Michael R Watts. 2014. An ultralow power athermal silicon modulator. Nature communications 5 (2014), 4008.

[58] Hsinyu Tsai, Stefano Ambrogio, Pritish Narayanan, Robert M Shelby, and Geoffrey W Burr. 2018. Recent progress in analog memory-based accelerators for deep learning. Journal of Physics D: Applied Physics 51, 28 (2018), 283001.

[59] International Communication Union. 2012. G.694.1 : Spectral grids for WDM applications: DWDM frequency grid. https://www.itu.int/rec/T-REC-G.694.1201202-I/en

[60] Dong Wang, Ke Xu, Qun Jia, and Soheil Ghiasi. 2019. ABM-SpConv: A Novel Approach to FPGA-Based Acceleration of Convolutional Neural Network Inference. In Proceedings of the 56th Annual Design Automation Conference 2019. ACM, 87.

[61] Chen Zhang, Peng Li, Guangyu Sun, Yijin Guan, Bingjun Xiao, and Jason Cong. 2015. Optimizing fpga-based accelerator design for deep convolutional neural networks. In Proceedings of the 2015 ACM/SIGDA International Symposium on Field-Programmable Gate Arrays. ACM, 161-170. 